\title{
Cuts for circular proofs
}

\author{
Jérôme Fortier ${ }^{1}$ and Luigi Santocanale ${ }^{2}$ \\ 1 LIF, AMU, Marseille, France \\ LaCIM, UQAM, Montréal, Canada \\ jerome.fortier@lif .univ-mrs.fr \\ 2 LIF, AMU, Marseille, France \\ luigi.santocanale@lif .univ-mrs.fr
}

One of the authors introduced in 2] a calculus of circular proofs for studying the computability arising from the following categorical operations: finite products and coproducts, initial algebras, final coalgebras. The calculus of [2] is cut-free; yet, even if sound and complete for provability, it lacks an important property for the semantics of proofs, namely fullness w.r.t. the class of natural categorical models called $\mu$-bicomplete category in $[3$.

We fix, with this work, this problem by adding the cut rule to the calculus. To this goal, we need to modify the syntactical constraints on the cycles of proofs so to ensure soundness of the calculus and at same time local termination of cut-elimination. The enhanced proof system fully represents arrows of the intended model, a free $\mu$-bicomplete category. We also describe a cut-elimination procedure as a model of computation arising from the above mentioned categorical operations. The procedure constructs a cut-free proof-tree with infinite branches out of a finite circular proof with cuts.

The calculus of circular proofs. Terms are constructed from a fixed set of variables $\mathbb{V}$ using the binary function symbols $\times,+$ and the constants 1,0 ; the set of terms will be denoted by TERMS. A directed system of equations is a tuple $S=\langle X, \tau, \pi\rangle$, where $X=\mathrm{BD}(S)$ is a finite subset of $\mathbb{V}, \tau: X \rightarrow$ TERMS, and $\pi: X \rightarrow \mathbb{N}$. FV $(S)$ shall denote the set of free variables of $S$, namely $\bigcup_{x \in X} \operatorname{VAR}(\tau(x)) \backslash \mathrm{BD}(S)$.

Intuitively, we think of the tuple $S$ as the system of equations $\left\{x=_{\theta(\pi(x))} \tau(x) \mid x \in X\right\}$ where $\theta(n)=\mu$ (least solution) if $n$ is odd and $\theta(n)=\nu$ (greatest solution) otherwise. The priority function $\pi$ also specifies the order by which we solve this system of equations. Given $n \geq 0$ and a system $S$, let $X_{n}=\{x \in \mathrm{BD}(S) \mid \pi(x) \leq n\}$ and let $S_{n}$ be the restriction of $S$ to $X_{n}$, namely $S_{n}=\left\langle X_{n}, \tau_{\uparrow X_{n}}, \pi_{\uparrow X_{n}}\right\rangle$. In particular, if $M=\max \{\pi(x) \mid x \in \operatorname{BD}(S)\}$, then we define $\operatorname{MAX}(S)=\{x \in \mathrm{BD}(S) \mid \pi(x)=M\}, \operatorname{LOW}(S)=X_{M-1}$, and let $P(S)$, the predecessor system, be $S_{M-1}$.

A sequent is a pair $(s, t)$ of terms, written as usual $s \vdash t$; SEQ shall denote the set of sequents. For a fixed directed system of equations $S$, the inference rules over $S$ are (instances of) the formal expressions appearing in Figure 1 1 . Let $\Sigma$ denote the set of justifications appearing on the right of these formal expressions. For a deterministic transition system $G$ over $\{0,1\}$ and $v \in G, \varsigma_{i} v$ shall denote the unique successor of $v$ labelled by $i$.

Definition 1. A pre-proof over $S$ is a tuple $\Pi=\langle G, \rho, \sigma\rangle$ where $G$ is a deterministic labelled digraph over the alphabet $\{0,1\}, \rho: G \rightarrow \Sigma$, and $\sigma=\left(\sigma_{\mathrm{L}}, \sigma_{\mathrm{R}}\right): G \rightarrow$ SEQ; moreover, for each $v \in G, \operatorname{outdeg}(v) \leq 2$ and

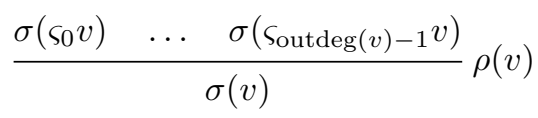

is an inference rule over $S$. 


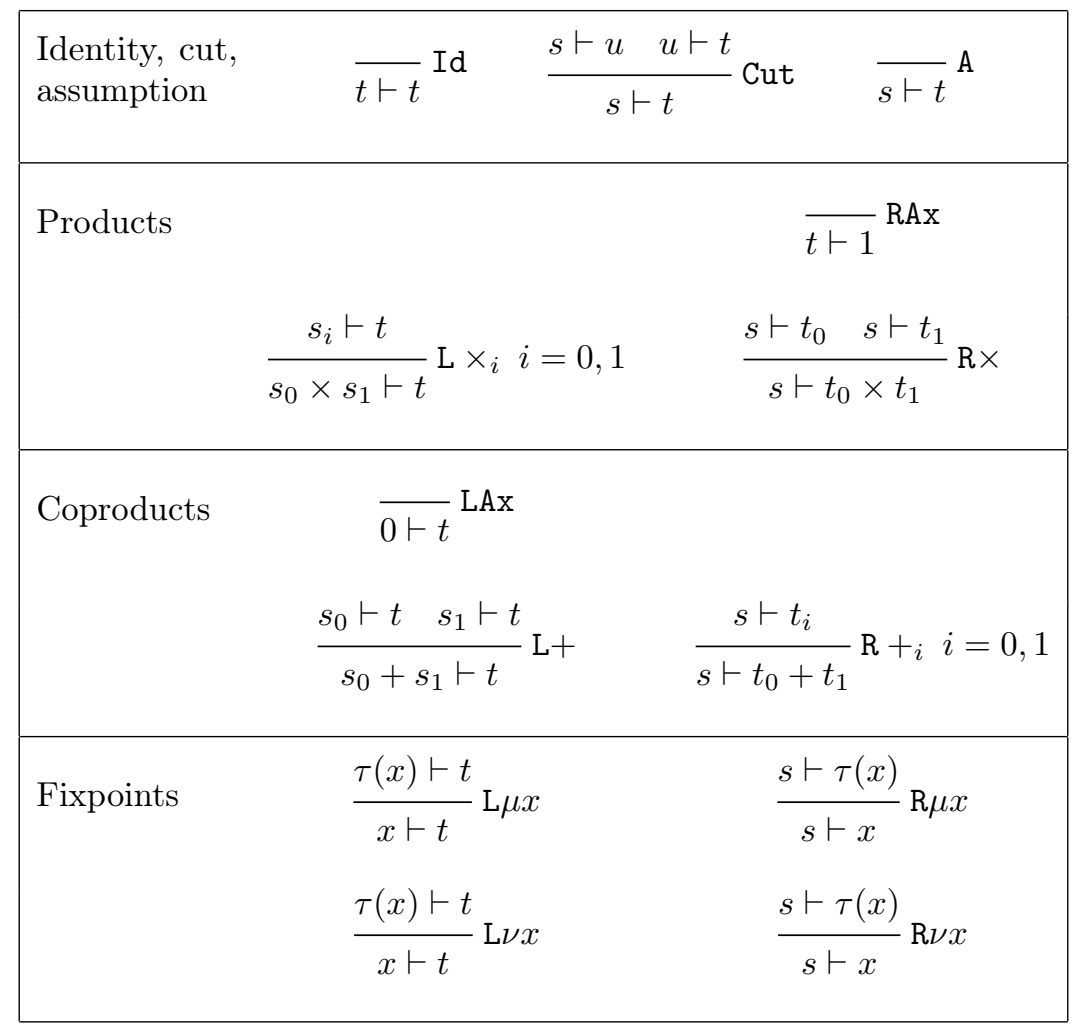

Figure 1: Inference rules of the system

A path $\Gamma$ of a pre-proof is left-traceable if, for all $n$, if $\rho(\Gamma(n))=$ Cut, then $\Gamma(n+1)=\varsigma_{0}(\Gamma(n))$; it is right-traceable if, for all $n$, if $\rho(\Gamma(n))=$ Cut, then $\Gamma(n+1)=\varsigma_{1}(\Gamma(n))$. $\Gamma$ has a left $\mu$-trace if $\Gamma$ is left-traceable, it contains a left regeneration rule, and the highest priority of its left regeneration rules is odd; $\Gamma$ has a right $\nu$-trace if if $\Gamma$ is right-traceable, it contains a right regeneration rule, and the highest priority of its right regeneration rules is even.

Definition 2. A circular proof is a pre-proof $\Pi=\langle G, \rho, \sigma\rangle$ such that every cycle in $G$ either has a left $\mu$-trace or a right $\nu$-trace.

Given a circular proof $\Pi$, we set $A_{\Pi}:=\{v \in G: \rho(v)=\mathrm{A}\}$ and $C_{\Pi}:=G \backslash A_{\Pi} ; A_{\Pi}$ is the set of assumptions of $\Pi$, while $C_{\Pi}$ is the set of its conclusions.

Semantics of the calculus. $\mu$-bicomplete categories were defined in 3 . Let $\mathcal{M}$ be a $\mu$ bicomplete category. Given $t \in$ TERMS and a finite subset $X$ with $\operatorname{VAR}(t) \subseteq X$, the natural semantics of $t$, denoted $|t|_{X}$, is a functor from $\mathcal{M}^{X}$ to $\mathcal{M}$. The formal definition of $|t|_{X}$ is by induction on the structure of $t$, as usual by interpreting the function symbols $1, \times, 0,+$ by means of the categorical structure. Given a directed system of equations $S$ and a finite subset $X$ such that $\mathrm{FV}(S) \subseteq X$ and $\operatorname{BD}(S) \cap X=\varnothing$, the semantics of $S$, noted by $\llbracket S \rrbracket_{X}$, is a functor from $\mathcal{M}^{X}$ to $\mathcal{M}^{\mathrm{BD}(S)}$. The definition is as follows:

Definition 3. If $\operatorname{BD}(S)=\varnothing$, then $\mathcal{M}^{\mathrm{BD}(S)}$ is the terminal category so that we let $\llbracket S \rrbracket_{X}$ be the unique functor from $\mathcal{M}^{X}$ to the terminal category. Otherwise, the predecessor system $P(S)$ is 
well-defined and its semantics is a functor from $\mathcal{M}^{X \cup \operatorname{MAX}(S)}$ to $\mathcal{M}^{\mathrm{BD}(P(S))}$. Let $G$ and $H$ be the functors so defined:

$$
\begin{aligned}
G & :=\left\langle|\tau(x)|_{[\mathrm{BD}(S) \cup X]} \mid x \in \operatorname{MAX}(S)\right\rangle: \mathcal{M}^{\mathrm{BD}(S) \cup X} \rightarrow \mathcal{M}^{\operatorname{MAX}(S)}, \\
H & :=\left\langle G, \llbracket P(S) \rrbracket_{\operatorname{MAX}(S) \cup X} \circ \operatorname{pr}_{\operatorname{MAX}(S) \cup X}^{\mathrm{BD}(S) \cup X},\right. \\
& \mathcal{M}^{\mathrm{BD}(S)} \times \mathcal{M}^{X}=\mathcal{M}^{\mathrm{BD}(S) \cup X} \longrightarrow \mathcal{M}^{\operatorname{MAX}(S)} \times \mathcal{M}^{\mathrm{BD}(P(S))}=\mathcal{M}^{\mathrm{BD}(S)} .
\end{aligned}
$$

If $\pi(\operatorname{MAX}(S))$ is odd, then $\llbracket S \rrbracket_{X}$ is the parametrized initial algebra of $H$; if $\pi(\operatorname{MAX}(S))$ is even, then $\llbracket S \rrbracket_{X}$ is the parametrized final coalgebra of $H$.

Finally, given a system $S$, a term $t$, and a finite subset $X$ with $\operatorname{FV}(S) \cup(\operatorname{VAR}(t) \backslash \operatorname{BD}(S)) \subseteq X$, the value of $t$ w.r.t. $S$, denoted $\llbracket t \rrbracket_{X}^{S}$, is the functor defined by:

$$
\llbracket t \rrbracket_{X}^{S}:=\left(\mathcal{M}^{X} \stackrel{\left\langle\mathrm{id}, \llbracket S \rrbracket_{X}\right\rangle}{\longrightarrow} \mathcal{M}^{X} \times \mathcal{M}^{\mathrm{BD}(S)}=\mathcal{M}^{X \cup \mathrm{BD}(S)} \stackrel{|t|_{X \cup \mathrm{BD}(S)}}{\longrightarrow} \mathcal{M}\right) .
$$

We shall use a sloppy notation and write just $\llbracket t \rrbracket$ in place of $\llbracket t \rrbracket_{X}^{S}$.

Lemma 4. For each $x \in \operatorname{BD}(S)$, if $\pi(x)$ is odd, then there exists a canonical invertible arrow $\zeta_{x}: \llbracket \tau(x) \rrbracket \rightarrow \llbracket x \rrbracket$; if $\pi(x)$ is even, then there exists a canonical invertible arrow $\xi_{x}: \llbracket x \rrbracket \rightarrow$ $\llbracket \tau(x) \rrbracket$.

With exception of Id and Cut, a rule Rule with assumptions $s_{i} \vdash t_{i}$ and conclusion $s \vdash t$ can be intertpreted as a natural transformation

$$
[\mathrm{Rule}]_{X, X^{\prime}}: \prod_{i=1, \ldots, n} \mathcal{M}\left(\llbracket s_{i} \rrbracket, \llbracket t_{i} \rrbracket\right) \rightarrow \mathcal{M}(\llbracket s \rrbracket, \llbracket t \rrbracket):\left(\mathcal{M}^{X}\right)^{o p} \times \mathcal{M}^{X} \rightarrow \text { Set } .
$$

(For the fixpoint rules, use the structure maps $\zeta_{x}, \zeta_{x}^{-1}, \xi_{x}, \xi_{x}^{-1}$ ). The above remark is almost true of Cut; if either we have a natural transformation $\beta: \llbracket u \rrbracket \rightarrow \llbracket t \rrbracket$, or a natural transformation $\gamma: \llbracket s \rrbracket \rightarrow \llbracket u \rrbracket$, then we have:

$$
[\text { Cut }, \beta]: \mathcal{M}(\llbracket s \rrbracket, \llbracket u \rrbracket) \rightarrow \mathcal{M}(\llbracket s \rrbracket, \llbracket t \rrbracket), \quad[\gamma, \text { Cut }]: \mathcal{M}(\llbracket u \rrbracket, \llbracket t \rrbracket) \rightarrow \mathcal{M}(\llbracket s \rrbracket, \llbracket t \rrbracket) .
$$

Definition 5. A circular proof $\Pi$ is homogeneous if it does not contain the rule Id and, for each $v \in \Pi$ with $\rho(v)=$ Cut, exactly one among $\varsigma_{0} v$ and $\varsigma_{1} v$ is an assumption of $\Pi$.

For $\Pi$ homogeneous, let $A_{\Pi}^{\mathrm{c}}=\left\{\varsigma_{i} v \in A_{\Pi} \mid \rho(v)=\right.$ Cut $\}$ and $A_{\Pi}^{\mathrm{s}}=\left\{\varsigma_{i} v \in A_{\Pi} \mid \rho(v) \neq\right.$ Cut $\}$; w.l.o.g., we shall assume that $A_{\Pi}^{\mathrm{c}} \cap A_{\Pi}^{\mathrm{s}}=\varnothing$. Given a collection of natural transformations $\beta=\left\{\beta^{v}: \llbracket \sigma_{\mathrm{L}}(v) \rrbracket \rightarrow \llbracket \sigma_{\mathrm{R}}(v) \rrbracket \mid v \in A_{\Pi}^{\mathrm{c}}\right\}$, the above rules give rise to a natural transformation

$$
\left[\Pi_{\beta}\right]: \prod_{v \in C_{\Pi}} \mathcal{M}\left(\llbracket \sigma_{\mathrm{L}}(v) \rrbracket, \llbracket \sigma_{\mathrm{R}}(v) \rrbracket\right) \times \prod_{v \in A_{\Pi}^{\mathrm{s}}} \mathcal{M}\left(\llbracket \sigma_{\mathrm{L}}(v) \rrbracket, \llbracket \sigma_{\mathrm{R}}(v) \rrbracket\right) \rightarrow \prod_{v \in C_{\Pi}} \mathcal{M}\left(\llbracket \sigma_{\mathrm{L}}(v) \rrbracket, \llbracket \sigma_{\mathrm{R}}(v) \rrbracket\right) .
$$

Theorem 6. For each system $S$, each homogeneous circular proof $\Pi$ over $S$, and each collection of natural transformations $\left\{\beta_{v}: \llbracket \sigma_{\mathrm{L}}(v) \rrbracket \rightarrow \llbracket \sigma_{\mathrm{R}}(v) \rrbracket \mid v \in A_{\Pi}^{c}\right\}$, there exists a unique natural transformation

$$
\left[\Pi_{\beta}\right]_{\dagger}: \prod_{v \in A_{\Pi}^{s}} \mathcal{M}\left(\llbracket \sigma_{\mathrm{L}}(v) \rrbracket, \llbracket \sigma_{\mathrm{R}}(v) \rrbracket\right) \longrightarrow \prod_{v \in C_{\Pi}} \mathcal{M}\left(\llbracket \sigma_{\mathrm{L}}(v) \rrbracket, \llbracket \sigma_{\mathrm{R}}(v) \rrbracket\right)
$$

satysfying the fixpoint equation $\left[\Pi_{\beta}\right]_{\dagger}=\left[\Pi_{\beta}\right] \circ\left\langle\left[\Pi_{\beta}\right]_{\dagger}, \mathrm{id}\right\rangle$. 
A circular proof is ground if it does not contain an assumption rule. A pointed circular proof is a pair $\langle\Pi, v\rangle$ where $\Pi$ is a ground circular proof and $v \in \Pi$. We can define $\llbracket \Pi, v \rrbracket$, the interpretation of $\langle\Pi, v\rangle$ with respect to the system $S$, by induction, almost as usual; the induction is now on the well-founded structure of maximal strongly connected components of the underlying graph of $\Pi$. To this goal the key observation is that if $\mathcal{C}$ is such a non trivial component of $\Pi$ (i.e. if there exists $v, u \in \mathcal{C}$ and a non-null path from $v$ to $u$ ), then the restriction of $\Pi$ to $\mathcal{C}$ is homogeneous. Thus Theorem 6 allows to interpret $\mathcal{C}$ as a sort of generalized inference rule, whose assumptions belong to strictly lesser components.

The calculus is full in this sense: if a pointed circular proof $\langle\Pi, v\rangle$ is such that $\sigma(v)=s[t / x] \vdash t$, then it is possible to construct a pointed circular proof $\left\langle\Pi^{\prime}, v^{\prime}\right\rangle$ whose semantics $\llbracket \Pi^{\prime}, v^{\prime} \rrbracket$ shall be the unique arrow $f$ such that $f \circ \zeta_{x}=\llbracket \Pi, v \rrbracket \circ \llbracket s \rrbracket(f)$. Of course, a dual property holds as well.

Cut elimination. We devise an algorithm that, given a pointed circular proof $\langle\Pi, v\rangle$, outputs a proof-tree which is cut-free, finitely branching but with possibly infinite branches. Just like in the classical case for Gentzen's system (see [1] for instance), the procedure consists in "pushing" every cut away from the root. Yet, this time, the output tree must be computed with a lazy (outermost) rather than eager (innermost) strategy. This is because not every path in $\Pi$ leads to a leaf, so that we have to eliminate cuts by performing a breadth-first search of $\Pi$. A main problem, see Figure 2 is that with this strategy it might be the case that we need to permute a cut with another cut. We dismiss this problem by merging consecutive cuts together in a sort of $n$-ary cut. Such an $n$-ary cut becomes the internal data structure (that we call a tape) of an automaton that tries to build up a branch of the the proof-tree. When the proof-tree branches, the automaton forks into several automata so to construct all the branches. Equivalently, we can think that the automaton undeterministically chooses which branch to construct. The automaton grows up the branch by means of commutative cut reductions at the extremities of the tape; if all the cuts in the tape are principal, the automaton undeterministically chooses one and reduces it, without constructing a new node on the branch. We can prove that the automaton does not perform "internal chatting". That is, the automaton eventually finds on its tape the oppurtunity to perfom a commutative cut reduction, thus growing the prefix of the proof-tree.

$$
\frac{t_{0} \vdash t_{1} \quad t_{1} \vdash t_{2}}{t_{0} \vdash t_{2}} \operatorname{Cut}_{t_{2} \vdash t_{3} \cdots t_{n-1} \vdash t_{n}} \mathrm{Cut} \Rightarrow \frac{t_{0} \vdash t_{1} \quad t_{1} \vdash t_{2} \quad \cdots \quad t_{n-1} \vdash t_{n}}{t_{0} \vdash t_{3}} \mathrm{Cut}
$$

Figure 2: Flattening cuts into a tape of cuts

\section{References}

[1] R. David, K. Nour, and C. Raffalli. Introduction à la logique, Théorie de la démonstration. Dunod, 2nd edition, 2004.

[2] Luigi Santocanale. A calculus of circular proofs and its categorical semantics. In Mogens Nielsen and Uffe Engberg, editors, FoSSaCS, volume 2303 of Lecture Notes in Computer Science, pages 357-371. Springer, 2002.

[3] Luigi Santocanale. $\mu$-bicomplete categories and parity games. Theoretical Informatics and Applications, 36:195-227, September 2002. 